\title{
Sample size calculations for the differential expression analysis of RNA-seq data using a negative binomial regression model
}

\author{
${ }^{1}$ Department of Bioinformatics and Biostatistics, School of Public Health and Information Sciences, University of Louisville, \\ Louisville, KY 40202, USA, E-mail: xolio013@louisville.edu. https://orcid.org/0000-0001-8345-9164. \\ ${ }^{2}$ Department of Anatomical Sciences and Neurobiology, School of Medicine, University of Louisville, Louisville, KY, USA, E- \\ mail:xolioo13@louisville.edu. https://orcid.org/0000-0001-8345-9164.
}

\begin{abstract}
:
High throughput RNA sequencing (RNA-seq) technology is increasingly used in disease-related biomarker studies. A negative binomial distribution has become the popular choice for modeling read counts of genes in RNA-seq data due to over-dispersed read counts. In this study, we propose two explicit sample size calculation methods for RNA-seq data using a negative binomial regression model. To derive these new sample size formulas, the common dispersion parameter and the size factor as an offset via a natural logarithm link function are incorporated. A two-sided Wald test statistic derived from the coefficient parameter is used for testing a single gene at a nominal significance level 0.05 and multiple genes at a false discovery rate 0.05 . The variance for the Wald test is computed from the variance-covariance matrix with the parameters estimated from the maximum likelihood estimates under the unrestricted and constrained scenarios. The performance and a sideby-side comparison of our new formulas with three existing methods with a Wald test, a likelihood ratio test or an exact test are evaluated via simulation studies. Since other methods are much computationally extensive, we recommend our M1 method for quick and direct estimation of sample sizes in an experimental design. Finally, we illustrate sample sizes estimation using an existing breast cancer RNA-seq data.
\end{abstract}

Keywords: a Wald test, differentially expressed genes (DEGs), power analysis, RNA-seq, sample size DOI: 10.1515/sagmb-2018-0021

\section{Introduction}

High-dimensional RNA-seq is a powerful tool for gene expression profiling and has been increasingly used for biomarker discovery and clinical studies (Wright et al., 2016; Xu et al., 2016). Differentially expressed genes (DEGs) or novel transcripts identified by RNA-seq serve as gene signatures for clinical diagnosis, prognosis, and estimating the efficacy of gene therapy or survival predictions (Rajan et al., 2014; Smith et al., 2015; Wright et al., 2015; Yao et al., 2015; Guo et al., 2016). With the rapid growth of clinical applications using RNA-seq, sample size estimation methods are critical for experiment design.

Since RNA-seq data are read counts, sample size calculations have been derived on the assumption of a Poisson distribution using the two-sided Wald, score or likelihood ratio tests (LRT) in the case of testing a single gene (Fang \& Cui, 2011; Li et al., 2013a). Alternatively, a sample size calculation derived from a score test was identical to that derived from a Wald test with parameters estimated from a maximum likelihood estimate under a null hypothesis $\left(H_{0}\right)$ between two conditions (Li et al., 2013a). Since thousands of genes per sample in RNA-seq data need to be tested simultaneously, a false discovery rate (FDR) for the multiple comparison was introduced in this study (Li et al., 2013a). However, the assumption of a Poisson distribution is inappropriate by the fact that the expected mean is equal to the variance, which does not usually hold for RNA-seq data. Studies have shown that the variance of read counts of a gene from RNA-seq data is significantly larger than its mean value, resulting in over-dispersed data (Anders \& Huber, 2010; Robinson \& Oshlack, 2010). Therefore, the variance needs to be adjusted for proper inference. Consequently, a negative binomial (NB) distribution with two parameters when comparing two groups has been widely used for modeling RNA-seq count data.

Recently, a sample size calculation method using a NB distribution and an exact test with the aid of edgeR was proposed, carrying out a two-sided test of single-gene or multiple-gene between two groups (Li, Su \& Shyr, 
2013b). Most recently, several sample size methods based on a NB distribution using well-known test statistics such as the Wald test, the log-transformed Wald test and the likelihood ratio test statistics with a one-sided test $p$-value were proposed ( $\mathrm{Li}$ et al., 2017). We noticed that sample size formulas derived from a Wald test in this paper were only based on one-sided test for the DEGs analysis which is a major limitation. For RNA-seq data, identification of the DEGs based on a fold change (FC) can be up (FC $\geq 1$ ) or down (FC $\leq 1)$. Therefore, a two-sided statistical test is more suitable. Besides, all sample size calculation methods derived from a negative binomial distribution for RNA-seq data assumed equal sample sizes (balanced designs) between two comparison groups. Although an imbalanced design is not common for RNA-seq studies, in some situations, especially in the clinical studies, it is preferred. For example, an RNA-seq study of breast cancer fusion transcripts contains forty-two Triple Negative Breast Cancer (TNBC) primary tumors and twenty-one uninvolved breast tissue samples that were adjacent to TNBC (Varley et al., 2014).

Furthermore, the variance for a Wald test used to derive the sample size calculations in the study (Li et al., 2017) was directly estimated from the variance formula in a NB distribution, in which the parameters of mean read counts were estimated by an unrestricted MLE or constrained MLE (CMLE) under $H_{0}$. But, the parameter space in the scenario of the constrained MLE is not restricted. However, studies of power and sample size calculations when comparing two groups in a NB distribution used another approach where the variance for a Wald test was estimated from a variance-covariance matrix (Aban, Cutter \& Mavinga, 2008; Zhu \& Lakkis, 2014). In these studies, Aban, Cutter, and Mavinga (2008) proposed a Wald test with a variance estimated from the variance-covariance matrix by comparing the two NB rates using the MRI Lesion count data. Further, Zhu et al. (Zhu \& Lakkis, 2014) proposed the explicit sample size calculation formulas based on a NB model, specifically applicable for modeling count data in asthma or chronic pulmonary disease (COPD).

In this paper, two explicit sample size calculation formulas based on the DEGs analysis for RNA-seq data are developed. For testing a single gene, we define a two-sided Wald test based on the standard GLM for comparing two treatment groups. The power equation and two sample size calculation formulas are derived from the Wald test where the variance is estimated from a variance-covariance matrix with the parameters estimated in two different scenarios. With this approach, our methods are able to incorporate the ratio of the size factor (geometric mean of normalization factors) for correcting RNA sequencing read depth and the fold change for the analysis of DEGs with a two-sided test statistic. The size factor in RNA-seq data is a parameter for a GLM, which is used for normalization of raw read counts. For testing multiple genes simultaneously in the DEG analysis, the Benjamini and Hochberg FDR is commonly used to adjust the $p$-values (Benjamini \& Hochberg, 1995) because the Bonferroni correction for a large number of tests is too conservative. An extended FDR correction was proposed for the microarray analysis (Storey, 2002) and subsequently used for developing sample calculations in RNA-seq (Li et al. 2013a and Li, Su, and Shyr 2013b). In this case, we derive the FDR-corrected sample size formulas by replacing $\alpha$ with $\alpha^{*}$ in the derived formulas. Moreover, a simulation study using a wide range of parameter settings was carried out to evaluate the proposed formulas under two scenarios versus the three existing methods for designing RNA-seq experiments. Of these three methods, the first one was derived from a Wald test with the parameters estimated under unrestricted terms, the second one was based on a log-likelihood ratio test (Li et al., 2017) and the third one was based on an exact test (Li, Su \& Shyr, 2013b). Finally, we illustrate how to estimate unequal or equal sample sizes using publicly accessible RNA-seq data on breast cancer. In summary, this study is motivated to develop new sample size calculation formulas using a standard GLM for RNA-seq data that are applicable in different scenarios, such as testing a single gene or multiple genes, imbalanced or balanced design, equal or unequal read depth across samples, or a two-sided test $p$-value for either down or up-regulated genes.

\section{Methods}

\subsection{Sample size formulas in the case of testing a single gene}

For a single gene in RNA-seq data, suppose that independent random sample $Y_{i j}$ from the $j$-th sample $(j=$ $\left.1, \ldots, n_{i}\right)$ and in the i-th treatment group $(i=0,1)$ follows a NB distribution. That is $Y_{i j} \sim N B\left(s_{i j} \gamma_{i}, \phi\right)$ or $Y_{i j} \sim N B\left(u_{i j}, \phi\right)$ (Robinson \& Smyth, 2008; Anders \& Huber, 2010), where $\gamma_{i}$ is the true gene expression level, $s_{i j}$ is a size factor to normalize the raw read count for the different read depth per sample, $u_{i j}=s_{i j} \gamma_{i}$ is the mean read count, and $\phi$ is a common dispersion parameter. Thus, $\left(Y_{i}=\sum_{j=1}^{n_{i}} Y_{i j} \sim N B\left(n_{i} \bar{s}_{i} \gamma_{i}, \phi / n_{i}\right)\right)$ or $N B\left(n_{i} u_{i}\right.$, $\left.\phi / n_{i}\right)$, where $\bar{s}_{i}=\frac{1}{n_{i}} \sum_{j=1}^{n_{i}} s_{i j}$ is the mean of the size factor for mapping reads in condition $i, u_{i}$ is the expected mean read counts for gene $g$ in group $i$ and $n_{i}$ (sample sizes) is the number of biological replicates with the 
assumption $k=n_{1} / n_{0}$. The probability mass function (PMF) of the observation $y_{i j}$ in a NB distribution is (Keene et al., 2007; Zhu \& Lakkis, 2014):

$$
\mathrm{P}\left(Y_{i j}=y_{i j}\right)=\frac{\Gamma\left(\phi^{-1}+y_{i j}\right)}{\Gamma\left(\phi^{-1}\right) y_{i j} !}\left(\frac{\phi u_{i j}}{1+\phi u_{i j}}\right)^{y_{i j}}\left(\frac{1}{1+\phi u_{i j}}\right)^{\phi^{-1}} .
$$

The above NB model utilizes the conventional parameterization called NB2 because we only know the mean read counts $\left(u_{i j}\right)$ of the gene, but the true expression $\left(\gamma_{i}\right)$ in the RNA-seq data is unknown (Mi, Di \& Schafer, 2015).

For a GLM, the true mean per size factor unit can be modeled by a log link function as:

$$
\log \gamma_{i}=b_{0}+b_{1} x_{i}
$$

where $\gamma_{i}=\frac{u_{i}}{\bar{s}_{i}}, \bar{s}_{i}$ denotes the mean of the size factors in group $i$, and $x_{i}$, the treatment group indicator for a single gene, takes value 0 if $i=0$ for control group and 1 if $i=1$ for treatment group. Thus, a ratio of the true expression $\gamma_{0}$ and $\gamma_{1}$ from equation (2) can be obtained by:

$$
\frac{\gamma_{1}}{\gamma_{0}}=e^{b_{1}}
$$

where $\gamma_{0}=e^{b_{0}}$ and $\gamma_{1}=e^{b_{0}+b_{1}}$

For the detection of a DEG from RNA-seq data, the ratio $\rho=\gamma_{1} / \gamma_{0}$ typically represents a fold change (FC). If it equals to one, this gene is considered to be not differentially expressed (Aban, Cutter \& Mavinga, 2008; Li et al., 2013a; Zhu \& Lakkis, 2014; Li et al., 2017). Therefore, testing the hypothesis of $\gamma_{i}$ is equivalent to test:

$$
H_{0}: b_{1}=0 \text { vs. } H_{1}: b_{1} \neq 0 \text {. }
$$

Replacing $u_{i j}=s_{i j} e^{b_{0}+b_{1} x_{i j}}$ in equations (1), the log-likelihood function is:

$$
l\left(\beta_{0}, \beta_{1}, \phi \mid y_{i j}, x_{i j}\right)=\sum_{i=0}^{1} \sum_{j=1}^{n_{i}}\left[\log \frac{\Gamma\left(\phi^{-1}+y_{i j}\right)}{\Gamma\left(\phi^{-1}\right) y_{i j} !}+y_{i j} \log \left(\phi s_{i j} e^{b_{0}+b_{1} x_{i j}}\right)-\left(y_{i j}+\frac{1}{\phi}\right) \log \left(1+\phi s_{i j} e^{b_{0}+b_{1} x_{i j}}\right)\right] .
$$

The model parameters $b_{0}, b_{1}$ and $\phi$ in equation (3) can be estimated using the maximum likelihood estimates (MLE), and the variance-covariance matrix of the model can be estimated via the inverse of the Fisher information matrix, asymptotically. Since the dispersion $\phi$ does not have a closed form, for simplicity, $\phi$ is treated as a known parameter for the two groups. Then, the first and second derivatives of log likelihood functions $(l)$ with respect to $b_{0}$ and $b_{1}$ from equation (3) are calculated, together with the Fisher information matrix, $I\left(b_{0}, b_{1}\right)$. Detailed procedures are given in Appendix A. Briefly, the Fisher information matrix can be expressed as:

$$
I\left(b_{0}, b_{1}\right)=\left(\begin{array}{cc}
A+B & B \\
B & B
\end{array}\right)
$$

where $A=\frac{n_{0} \bar{s}_{0} e^{b_{0}}}{1+\phi \bar{s}_{0} e^{b_{0}}}$ and $B=\frac{n_{1} \bar{s}_{e} e^{b_{0}+b_{1}}}{1+\phi \bar{s}_{1} e^{b_{0}+b_{1}}}$.

Let $\left(\hat{b}_{0}, \hat{b}_{1}\right)^{\prime}$ be the MLEs of model parameters $\left(b_{0}, b_{1}\right)^{\prime}$. The asymptotic normality and consistency of $\left(\hat{b}_{0}, \hat{b}_{1}\right)^{\prime}$ follow the large-sample properties of MLE; and the variance-covariance matrix of $\left(\hat{b}_{0}, \hat{b}_{1}\right)^{\prime}$ is the inverse of information matrix of $I_{n_{0}, n_{1}}\left(b_{0}, b_{1}\right)$ asymptotically. Thus, the variance of $\hat{b}_{1}$ is approximately:

$$
\operatorname{Var}\left(\hat{b}_{1}\right)=\frac{1}{n_{0}}\left(\frac{1+\phi \bar{s}_{0} e^{b_{0}}}{\bar{s}_{0} e^{b_{0}}}+\frac{1+\phi \bar{s}_{1} e^{b_{0}+b_{1}}}{k \bar{s}_{1} e^{b_{0}+b_{1}}}\right)
$$

where $k=n_{1} / n_{0}$. Replaced $\gamma_{0}=e^{b_{0}}, \gamma_{1}=e^{b_{0}+b_{1}}, \rho=\frac{\gamma_{1}}{\gamma_{0}}=e^{b_{1}}, w=\frac{\bar{s}_{1}}{\bar{s}_{0}}, u_{0}=\bar{s}_{0} \gamma_{0}$ and $u_{1}=\bar{s}_{1} \gamma_{1}=w \rho u_{0}$ in (4), the variance of $\hat{b}_{1}$ becomes: 


$$
\operatorname{Var}\left(\hat{b}_{1}\right)=\frac{1}{n_{0}}\left[\frac{1}{u_{0}}\left(1+\frac{1}{k w \rho}\right)+\left(1+\frac{1}{k}\right) \phi\right]=\frac{V_{i}}{n_{0}}
$$

where, $i=0$ when the parameter $u_{0}$ is estimated under $H_{0}$, denoted by $\tilde{u}_{0}$; and $i=1$ when the parameter $u_{0}$ is estimated under $H_{1}$, assuming the true mean for the control group.

To test the null hypothesis $H_{0}: b_{1}=0, \hat{b}_{1}$ asymptotically follows a normal distribution with mean $\log (\rho)=$ $\log \left(\frac{\gamma_{1}}{\gamma_{0}}\right)$ and variance $V_{0}\left(\hat{b}_{1}\right)=V_{0} / n_{0}$ with $\rho=1$ under $H_{0}$. The two-sided Wald test is to reject $H_{0}$ if:

$$
\left|Z_{w}\right|=\frac{\left|\hat{b}_{1}\right|}{\sqrt{V_{0} / n_{0}}}>Z_{1-\frac{\alpha}{2}}
$$

where $V_{0}$ is the corresponding variance proportion estimated in (5) under $H_{0}$ :

$$
V_{0}=\frac{1}{\tilde{u}_{0}}\left(1+\frac{1}{k w \rho}\right)+\left(1+\frac{1}{k}\right) \phi,
$$

and $\tilde{u}_{0}$ is the estimated mean read counts from the MLE under $H_{0}$.

Thus, a power of $(1-\beta)$ is obtained under the alternative hypothesis $H_{1}: b_{1} \neq 0$. When $\left|Z_{w}\right|>Z_{1-\alpha / 2}$, an approximately two-sided Wald test of $\hat{b}_{1}$ with an expected mean $\log (\rho)=\log \left(\frac{\gamma_{1}}{\gamma_{0}}\right)$ and variance $V_{1}\left(\hat{b}_{1}\right)=V_{1} / n_{0}$ is expressed as:

$$
\frac{\left|\hat{b}_{1}-E\left(\hat{b}_{1}\right)\right|}{\sqrt{V_{1} / n_{0}}}>\left[\frac{Z_{1-\alpha / 2} \sqrt{V_{0} / n_{0}}-\log \left(\frac{\gamma_{1}}{\gamma_{0}}\right)}{\sqrt{V_{1} / n_{0}}}\right],
$$

where $V_{1}$ is the corresponding variance proportion estimated in (5) under $H_{1}$ :

$$
V_{1}=\frac{1}{u_{0}}\left(1+\frac{1}{k w \rho}\right)+\left(1+\frac{1}{k}\right) \phi,
$$

and $u_{0}$ is the true mean read counts estimated from the MLE under $H_{1}$.

For a specific alternative hypothesis $H_{1}: b_{1}>0$, the approximate power of the test can be expressed as:

$$
1-\beta=1-\Phi\left[\frac{Z_{1-\alpha / 2} \sqrt{V_{0} / n_{0}}-\log \left(\frac{\gamma_{1}}{\gamma_{0}}\right)}{\sqrt{V_{1} / n_{0}}}\right] .
$$

Solving equation (8), we obtain the sample size formula as:

$$
n_{0}=\frac{\left(Z_{1-\alpha / 2} \sqrt{V_{0}}+Z_{1-\beta} \sqrt{V_{1}}\right)^{2}}{\{\log (\rho)\}^{2}},
$$

where $Z_{p}$ is the quantile of the standard normal distribution, $\rho$ is a FC under $H_{1}$. When the alternative hypothesis $H_{1}$ is $b_{1}<0$, equation (9) is also true. This approach for estimation of sample size with a two-sided test is referred to the previous studies on RNA-seq (Fang \& Cui, 2011; Li et al., 2013a; Li, Su \& Shyr, 2013b). As for the variance estimations under $H_{0}$, the mean read counts $\tilde{u}_{0}$ in the control group in (7) can be estimated through the following five approaches.

\section{Method 1 (M1): Use the MLE under $\mathbf{H}_{1}$}

Let $\tilde{u}_{0}$ in $V_{0}$ equal to the true mean $u_{0}$ in the control group, and $\tilde{u}_{1}$ equal to the true mean $\rho w u_{0}$ in the treatment group, which is equivalent to $\tilde{\gamma}_{0}=\gamma_{0}$ and $\tilde{\gamma}_{1}=\gamma_{1}=\rho \gamma_{0}$. Then, we have $V_{0}=V_{1}$ in this scenario. Thus, the sample size formula in equation (8) becomes:

$$
n_{M 1}=\frac{\left(Z_{1-\alpha / 2}+Z_{1-\beta}\right)^{2} V_{1}}{\{\log (\rho)\}^{2}},
$$


where $V_{1}=\frac{1}{u_{0}}\left(1+\frac{1}{k w \rho}\right)+\left(1+\frac{1}{k}\right) \phi=\frac{1}{\bar{y}_{0}}\left(1+\frac{1}{k w \rho}\right)+\left(1+\frac{1}{k}\right) \phi$. The parameter $u_{0}$ can be estimated by the unrestricted MLE in Appendix B similar to the method discussed by Li et al. (Li et al., 2017). Thus, we obtain $u_{0}=\hat{u}_{0}=\bar{y}_{0}$ and $u_{1}=\hat{u}_{1}=\rho w \hat{u}_{0}$, where $\bar{y}_{0}$ is the sample mean estimated from the DEGs in the control group (Aban, Cutter \& Mavinga, 2008; Li et al., 2017). This method for sample size estimation is written in R (v3.5.1) with the source codes available in the supplementary file SampleSize_supportInfo.R.

Method 2 (M2): Use the MLE under $\mathrm{H}_{0}$

Under $H_{0}: \rho=\frac{\gamma_{1}}{\gamma_{0}}=1$, we first estimate $\tilde{\gamma}_{0}$ and $\tilde{u}_{0}$ using the MLE (Appendix B):

$$
\begin{array}{r}
\tilde{\gamma}_{0}=\frac{2(1+k w \rho) \gamma_{0}}{(1+k)(1+w)}, \\
\tilde{u}_{0}=\bar{s}_{0} \tilde{\gamma}_{0}=\frac{2(1+k w \rho) u_{0}}{(1+k)(1+w)},
\end{array}
$$

where $u_{0}$, the true mean read counts, is estimated from the sample mean in the control group discussed in the M1 method. Then, we have $V_{0}=V_{0 M 2}=\frac{1}{\tilde{u}_{0}}\left(1+\frac{1}{k w \rho}\right)+\left(1+\frac{1}{k}\right) \phi$. Thus, the sample size formula under $V_{0}=V_{0 M 2}$ in (9) becomes:

$$
n_{M 2}=\frac{\left(Z_{1-\alpha / 2} \sqrt{V_{0 M 2}}+Z_{1-\beta} \sqrt{V_{1}}\right)^{2}}{\{\log (\rho)\}^{2}} .
$$

\section{Method 3 (M3)}

The method 3 (M3) is based on an existing method for an equal sample size calculation with a one-sided Wald test recently proposed by $\mathrm{Li}$ et al. (2017). The variance of a Wald test was directly estimated from the variance formula in a NB distribution with the parameter estimated under the unrestricted MLE. For a twosided Wald test, it is:

$$
n_{M 3}=\frac{\left(1+\frac{\rho}{w}+\phi \rho^{2} u_{0}+\phi u_{0}\right)\left(Z_{1-\alpha / 2}+Z_{1-\beta}\right)^{2}}{u_{0}(\rho-1)^{2}},
$$

where $Z_{1-\alpha}$ in the existing method is replaced by $Z_{1-\alpha / 2}$ in the M3 method.

\section{Method 4 (M4) and Method 5 (M5)}

The M4 and M5 methods are the public methods with the equal sample size calculations derived from a likelihood ratio test (Li et al., 2017) and an exact test (Li, Su \& Shyr, 2013b), respectively. Both M4 and M5 used a similar approach to define a power function in order to compute the sample size for a balanced experiment design. Briefly, a power function was defined via a joint probability between two negative binomial mean reads and expressed as:

$$
\operatorname{Pr}\left(n, \rho, u_{0}, \phi, w, \alpha\right)=\sum_{x_{0}=0}^{\infty} \sum_{x_{1}=0}^{\infty} f\left(n w \rho u_{0}, \frac{\phi}{n}\right) f\left(n u_{0}, \frac{\phi}{n}\right) I\left(P\left(Y_{0}=y_{0}, Y_{1}=y_{1}\right)<\alpha\right),
$$

where $I(\cdot)$ denotes the indicator function of $p$-value. In M4, the $p$-value in equation (13) is obtained from a twosided log-likelihood ratio test with a $\chi_{1}^{2}$ distribution and the $p$-value in M5 was computed using an exact test with the aid of edgeR (Li, Su \& Shyr, 2013b; Robinson \& Oshlack, 2010). Thus, given a nominal or desired power $1-\beta$, the power of the test is a function of the sample size $n$ in the form of

$$
1-\beta=\operatorname{Pr}\left(n, \rho, u_{0}, \phi, w, \alpha\right) .
$$

Therefore, the required sample size $n$ at a nominal power $1-\beta$ and a significance level a can be computed by solving equation (14) through a numerical approach with respect to $n$.

\subsection{Sample size formulas in testing multiple genes simultaneously}

The sample size formulas for a single gene are defined in equations (14-12), where $\alpha$ is the significance level and $(1-\beta)$ is the power in percentile. In an RNA-seq experiment, thousands of genes need to be tested simultaneously for DEGs analysis between groups. In this case, the sample size formulas for a single gene need to be 
adjusted due to multiple testing problems. In this section, we derive sample size calculations by incorporating FDR controlling based on the previous studies (Li et al., 2013a; Li, Su \& Shyr, 2013b; Li et al., 2017). Briefly, given the control false discovery rate (FDR) at a specified level $f$, the adjusted and smaller type I error level of $\alpha^{*}$ for the expected number of true rejection $r_{1}$ is:

$$
\alpha^{*}=\frac{r_{1} f}{m_{0}(1-f)},
$$

where $m_{0}$ is the number of true null hypotheses, $r_{1}=E\left(M_{\mathrm{t}}\right)=E\left(M-M_{0}\right)$ is the expected number of true rejections, $M_{0}$ is the number of false discoveries, and $M$ is the total number of genes declared significance, and $f$ is the control FDR at a specified level. Replacing the $\alpha$ in equations (10-12) with an $\alpha^{*}(15)$, the corresponding sample size calculation formulas corrected by FDR at level $f$ are, respectively:

$$
\begin{gathered}
n_{M 1^{*}}=\frac{\left(Z_{1-\alpha^{*} / 2}+Z_{1-\beta}\right)^{2} V_{1}}{\{\log (\rho)\}^{2}}, \\
n_{M 2^{*}}=\frac{\left(Z_{1-\alpha^{*} / 2} \sqrt{V_{0 M 2}}+Z_{1-\beta} \sqrt{V_{1}}\right)^{2}}{\{\log (\rho)\}^{2}}, \\
n_{M 3}=\frac{\left(1+\frac{\rho}{w}+\phi \rho^{2} u_{0}+\phi u_{0}\right)\left(Z_{1-\alpha * / 2}+Z_{1-\beta}\right)^{2}}{u_{0}(\rho-1)^{2}} .
\end{gathered}
$$

Similarly, replacing $a$ with $\alpha^{*}$ in equation (14), we obtain the function with respect to $n$ as

$$
1-\beta=r\left(n, \rho, u_{0}, \phi, w, \alpha^{*}\right) .
$$

Thus, by solving (19) numerically, the sample size for controlling FDR at level $f$ can be obtained for M4 and M5.

\section{Simulation for performance evaluation}

Simulation studies are conducted to evaluate the performance of the proposed sample size calculation methods and the existing methods. It includes two parts: in the first part, equal sample sizes and power are estimated in the case of testing a single gene; in the second part, sample sizes and power are estimated in the case of testing multiple genes under a balanced or imbalanced experimental design with an equal or unequal read depth. The $\mathrm{R}$ codes are available upon request to the first author.

\subsection{Sample size and power calculations in the case of testing a single gene}

In this simulation, the hypothesis test for giving a single gene is that the gene is not differentially expressed when the FC $\left(\rho=\frac{\gamma_{1}}{\gamma_{0}}\right)$ is equal to one, which is equivalent to $b_{1}=0$ in a GLM. In an alternative, the gene is considered differentially expressed when the FC is different from 1 . Hence, FC is set to be $0.5,0.7,1.5,2$ or 3 , corresponding to $b_{1} \neq 0$. The mean read count of the DEGs in the control group $u_{0}$ is set to be $1,5,10$ or 20 . The ratio of mean size factors $w=\frac{\bar{s}_{1}}{\bar{s}_{0}}$ is set to be 1 for an equal read depth, or 1.2 for an unequal read depth; the constant dispersion parameter is set to be $0.1,0.5$ or 0.7 ; and the ratio of sample sizes is set to be $k=\frac{n_{1}}{n_{0}}=1$ or $\frac{3}{2}$, where $k=1$ is for a balanced design.

For each combination of these parameter settings, sample sizes for testing a single gene are calculated using our formulas (M1 and M2) in (10-11) and three existing methods (M3 to M5) in (12-14), given an 80\% nominal power at the significance level of $\alpha=0.05$. Given the estimated sample size, the corresponding powers are assessed for all the methods via the simulation studies.

Briefly, given the estimated sample size and each designed parameter setting, two sets of NB random variables are generated for two groups: the control and the experimental group using an R script. For the control 
group, the random samples are generated given the parameters $n_{0}, u_{0}$ and $\phi$. For the treatment group, the random samples are generated given the parameters $n_{1}=k n_{0}, u_{1}=\rho w u_{0}$ and $\phi$. The Wald test for our new methods is used for testing the significance using the coefficient $b_{1}$ estimated from the MLE $\hat{b}_{1}=\frac{\bar{y}_{1}}{w \bar{y}_{0}}$ and the variance estimated from (7) under the two different approaches. The FC is considered significantly different when the two-sided $p$-value $\leq 0.025$. We have repeated the procedure 5000 times, and the power is calculated as the percentage of the number of times that the $H_{0}$ is rejected in the 5000 simulated data. The results are listed in Table $1(w=1$ and $k=1)$, and supplementary Tables $\mathrm{S} 1(w=1.2$ and $k=1)$ and S2 $(w=1$ and $k=3 / 2)$ as Support Information.

Table 1: Equal sample sizes $\left(n_{0}\right)$ and simulated power in parentheses from five methods.

\begin{tabular}{|c|c|c|c|c|c|c|c|}
\hline$\rho$ & $\phi$ & $u_{0}$ & M1 & M2 & M3 & M4 & M5 \\
\hline \multirow[t]{12}{*}{0.5} & 0.1 & 1 & $52(0.78)$ & $63(0.77)$ & $51(0.81)$ & $49(0.80)$ & $54(0.81)$ \\
\hline & & 5 & $13(0.79)$ & $15(0.79)$ & $13(0.81)$ & $13(0.82)$ & $14(0.81)$ \\
\hline & & 10 & $8(0.78)$ & $9(0.77)$ & $9(0.84)$ & $8(0.81)$ & $9(0.83)$ \\
\hline & & 20 & $6(0.81)$ & $6(0.77)$ & $6(0.81)$ & $6(0.83)$ & $6(0.81)$ \\
\hline & 0.5 & 1 & $65(0.78)$ & $77(0.78)$ & $67(0.81)$ & $61(0.79)$ & $68(0.80)$ \\
\hline & & 5 & $26(0.79)$ & $28(0.78)$ & $29(0.83)$ & $26(0.80)$ & $27(0.81)$ \\
\hline & & 10 & $21(0.78)$ & $22(0.78)$ & $24(0.83)$ & $22(0.81)$ & $22(0.80)$ \\
\hline & & 20 & $19(0.80)$ & $19(0.79)$ & $22(0.84)$ & $19(0.80)$ & $20(0.82)$ \\
\hline & 0.7 & 1 & $72(0.78)$ & $83(0.78)$ & $75(0.82)$ & $69(0.80)$ & $74(0.80)$ \\
\hline & & 5 & $33(0.79)$ & $35(0.79)$ & $37(0.83)$ & $33(0.80)$ & $34(0.80)$ \\
\hline & & 10 & $28(0.80)$ & $29(0.79)$ & $32(0.84)$ & $28(0.81)$ & $29(0.82)$ \\
\hline & & 20 & $25(0.79)$ & $26(0.80)$ & $30(0.85)$ & $26(0.81)$ & $26(0.81)$ \\
\hline \multirow[t]{12}{*}{0.7} & 0.1 & 1 & $162(0.80)$ & $180(0.80)$ & $161(0.81)$ & $162(0.81)$ & $168(0.81)$ \\
\hline & & 5 & $42(0.78)$ & $46(0.79)$ & $43(0.80)$ & $42(0.80)$ & $44(0.80)$ \\
\hline & & 10 & $27(0.78)$ & $29(0.80)$ & $28(0.80)$ & $28(0.81)$ & $28(0.80)$ \\
\hline & & 20 & $20(0.79)$ & $21(0.80)$ & $20(0.79)$ & $20(0.80)$ & $20(0.81)$ \\
\hline & 0.5 & 1 & $212(0.79)$ & $230(0.79)$ & $213(0.80)$ & $209(0.80)$ & $217(0.80)$ \\
\hline & & 5 & $92(0.80)$ & $95(0.79)$ & $95(0.81)$ & $91(0.80)$ & $94(0.81)$ \\
\hline & & 10 & $77(0.79)$ & $79(0.80)$ & $80(0.81)$ & $77(0.80)$ & $78(0.80)$ \\
\hline & & 20 & $69(0.80)$ & $70(0.80)$ & $72(0.81)$ & $70(0.80)$ & $71(0.81)$ \\
\hline & 0.7 & 1 & $236(0.79)$ & $255(0.80)$ & $239(0.81)$ & $235(0.81)$ & $243(0.81)$ \\
\hline & & 5 & $116(0.80)$ & $120(0.80)$ & $121(0.81)$ & $117(0.80)$ & $119(0.80)$ \\
\hline & & 10 & $101(0.80)$ & $103(0.79)$ & $106(0.82)$ & $102(0.81)$ & $103(0.81)$ \\
\hline & & 20 & $94(0.81)$ & $95(0.80)$ & $98(0.82)$ & $95(0.82)$ & $95(0.82)$ \\
\hline \multirow[t]{12}{*}{1.5} & 0.1 & 1 & $89(0.81)$ & $78(0.81)$ & $89(0.80)$ & $93(0.82)$ & $92(0.81)$ \\
\hline & & 5 & $25(0.81)$ & $23(0.82)$ & $26(0.81)$ & $28(0.84)$ & $27(0.82)$ \\
\hline & & 10 & $18(0.82)$ & $16(0.80)$ & $18(0.81)$ & $19(0.84)$ & $18(0.81)$ \\
\hline & & 20 & $14(0.82)$ & $13(0.81)$ & $14(0.81)$ & $15(0.84)$ & $14(0.81)$ \\
\hline & 0.5 & 1 & $127(0.81)$ & $116(0.81)$ & $130(0.81)$ & $133(0.81)$ & $131(0.80)$ \\
\hline & & 5 & $64(0.81)$ & $61(0.80)$ & $67(0.81)$ & $69(0.83)$ & $65(0.80)$ \\
\hline & & 10 & $56(0.81)$ & $55(0.81)$ & $59(0.82)$ & $60(0.83)$ & $57(0.81)$ \\
\hline & & 20 & $52(0.81)$ & $51(0.81)$ & $55(0.82)$ & $56(0.83)$ & $53(0.81)$ \\
\hline & 0.7 & 1 & $146(0.81)$ & $135(0.81)$ & $150(0.81)$ & $153(0.82)$ & $150(0.80)$ \\
\hline & & 5 & $83(0.80)$ & $81(0.80)$ & $87(0.81)$ & $89(0.82)$ & $85(0.80)$ \\
\hline & & 10 & $75(0.80)$ & $74(0.81)$ & $79(0.81)$ & $81(0.83)$ & $76(0.80)$ \\
\hline & & 20 & $71(0.80)$ & $70(0.80)$ & $75(0.81)$ & $77(0.82)$ & $72(0.80)$ \\
\hline \multirow[t]{12}{*}{2} & 0.1 & 1 & $28(0.84)$ & $22(0.84)$ & $27(0.81)$ & $28(0.83)$ & $29(0.82)$ \\
\hline & & 5 & $8(0.82)$ & $7(0.81)$ & $9(0.82)$ & $9(0.84)$ & $9(0.82)$ \\
\hline & & 10 & $6(0.83)$ & $5(0.79)$ & $6(0.81)$ & $6(0.83)$ & $6(0.81)$ \\
\hline & & 20 & $4(0.76)$ & $4(0.79)$ & $5(0.82)$ & $5(0.84)$ & $5(0.83)$ \\
\hline & 0.5 & 1 & $41(0.82)$ & $35(0.81)$ & $43(0.82)$ & $43(0.83)$ & $42(0.80)$ \\
\hline & & 5 & $21(0.81)$ & $20(0.81)$ & $24(0.84)$ & $23(0.84)$ & $22(0.81)$ \\
\hline & & 10 & $19(0.81)$ & $18(0.80)$ & $22(0.84)$ & $21(0.85)$ & $20(0.82)$ \\
\hline & & 20 & $18(0.81)$ & $17(0.79)$ & $21(0.85)$ & $19(0.83)$ & $18(0.81)$ \\
\hline & 0.7 & 1 & $47(0.82)$ & $42(0.82)$ & $51(0.83)$ & $50(0.83)$ & $49(0.81)$ \\
\hline & & 5 & $28(0.80)$ & $27(0.80)$ & $32(0.83)$ & $30(0.82)$ & $29(0.80)$ \\
\hline & & 10 & $25(0.80)$ & $25(0.81)$ & $30(0.85)$ & $28(0.84)$ & $26(.81)$ \\
\hline & & 20 & $24(.80)$ & $24(0.80)$ & $29(0.85)$ & $26(0.83)$ & $25(0.81)$ \\
\hline \multirow[t]{4}{*}{3} & 0.1 & 1 & $10(0.87)$ & $7(0.86)$ & $10(0.84)$ & $9(0.81)$ & $10(0.81)$ \\
\hline & & 5 & $3(0.84)$ & $2(0.77)$ & $4(0.90)$ & $4(0.91)$ & $4(0.89)$ \\
\hline & & 10 & $2(0.79)$ & $2(0.84)$ & $3(0.90)$ & $3(0.92)$ & $3(0.91)$ \\
\hline & & 20 & $2(0.86)$ & $2(0.89)$ & $2(0.82)$ & $2(0.86)$ & $2(0.85)$ \\
\hline
\end{tabular}




\begin{tabular}{rrrrrrr}
0.5 & 1 & $15(0.84)$ & $12(0.82)$ & $18(0.86)$ & $16(0.85)$ & $16(0.83)$ \\
& 5 & $8(0.78)$ & $8(0.82)$ & $11(0.87)$ & $9(0.84)$ & $9(0.82)$ \\
& 10 & $7(0.77)$ & $7(0.79)$ & $11(0.90)$ & $8(0.82)$ & $8(0.81)$ \\
0.7 & 20 & $7(0.80)$ & $7(0.81)$ & $10(0.90)$ & $8(0.85)$ & $8(0.81)$ \\
& 1 & $18(0.83)$ & $15(0.83)$ & $22(0.87)$ & $18(0.83)$ & $19(0.85)$ \\
& 5 & $11(0.82)$ & $10(0.79)$ & $15(0.88)$ & $12(0.84)$ & $12(0.83)$ \\
& 10 & $10(0.80)$ & $10(0.81)$ & $15(0.91)$ & $11(0.83)$ & $11(0.82)$ \\
& 20 & $10(0.81)$ & $9(0.77)$ & $14(0.89)$ & $11(0.84)$ & $10(0.80)$ \\
\hline
\end{tabular}

The parameter values are: fold change $(\rho)$, dispersion $(\phi)$ and mean reads in the control group $\left(u_{0}\right)$ given $w=1, \alpha=0.05$ and an $80 \%$ nominal power. M1 and M2 are our methods.

A similar approach is used to estimate the corresponding power for the existing methods (M3-M5) for testing the significance of the FC. Briefly, for the M3 and M4 methods, a two-sided test was conducted by replacing the significance $\alpha$ with $\alpha / 2$. The simulated power for the M5 method is estimated using an exact test with the R codes similar to Li et al. (Li, Su \& Shyr, 2013b). The results are listed in Table 1 and S1 (Support Information in a supplementary file).

The results in Table 1,S1 and S2 illustrate the sample size and the simulated power in parentheses with the combinational settings in the case of testing a single gene. We first examined the changing pattern of sample sizes with different values of $u_{0}, \phi$ and $\rho$ for the five methods. We observed that the sample size $n$ decreases in all five methods as read count $u_{0}$ increases from 1 to 20 , given a fixed $\rho$ and $\phi$ or vice versa, as expected. This pattern suggests that a larger sample size for a lowly expressed gene is required in order to achieve an empirical power close to $80 \%$ in the DEG analysis. Given fixed $u_{0}$ and $\rho$, the sample size increases as $\phi$ increases. This is also expected due to higher variation of genes across samples. Moreover, given a fixed $\phi$ and $u_{0}$, the sample size decreases as $\rho$ increases. These results suggest that a smaller $n$ is required for a larger fold change of a gene between two groups.

We further examine additional simulation studies to assess the ratio of the size factor $w=1.2$ on accuracy of the sample sizes to help understanding the necessity of extension from the method proposed by Zhu et al. (Zhu \& Lakkis, 2014). The results in Table S1 show that the $\mathrm{n}$ decreases in all the methods as w increases from $w$ $=1$ (Table 1 ) to 1.2 (Table S1) given a fixed $u_{0}=1$ or $5, \rho$ and $\phi$. However, when $u_{0}=10$ or $20, \mathrm{n}$ is either the same or slightly changed when replacing $w=1$ with $w=1.2$. This observation suggests that the read depth can affect sample size when the gene has a mean read counts in the control group smaller than 10. Further, the results in Table S2 with the settings of $k=3 / 2$ and $w=1$ show that a smaller sample size in the control group is required compared with a balanced design in Table 1 as expected. But a larger sample size for the treatment group is required in these settings.

Most importantly, we focused on the comparison of our new methods with the existing methods using two approaches. In the first approach, we examined n given an $80 \%$ nominal power and significance level of $\alpha=0.05$. Overall, the sample size $n$ estimated from the two new methods and the existing methods varies in different parameter settings (Table 1 and S1). We assumed that the better method would require a smaller sample size to achieve an actual power close to the desired power. To do this, we examined the $\mathrm{n}$ based on $\mathrm{FC}<1$ and FC $>1$ (Table 1). In the case of FC $<1$, we observed that M1 in a GLM under the first scenario, and M4, with a likelihood ratio test, achieve a slightly smaller $\mathrm{n}$ than the other methods in most settings. In the case of FC $>1$, we observed that M2 achieves a smaller $\mathrm{n}$ than the other methods in almost all settings. Although $\mathrm{n}$ required from M1 is larger than M2, it is smaller than the three existing methods in many settings (Table 1). The existing M3 performs worst compared to other methods.

Table 2: The $p$-values obtained from a paired Wilcox test and t-test.

\begin{tabular}{crrrr}
\hline Wilcox test & M2 & M3 & M4 & M5 \\
\hline M1 & 0.6301 & 0.0004 & 0.0051 & 0.3431 \\
M2 & - & 0.0001 & 0.0017 & 0.6155 \\
M3 & & - & 0.4086 & $<0.0001$ \\
M4 & & & - & 0.0007 \\
T test & 0.8094 & 0.0002 & 0.0044 & 0.8746 \\
M1 & - & 0.0001 & 0.0031 & 0.9591 \\
M2 & & - & 0.1829 & 0.0003 \\
M3 & & - & 0.0069 \\
M4 & & - & \\
\hline
\end{tabular}

The test statistics are used for testing the significance given the absolute value of difference between simulated power and an $80 \%$ desired power. 
Next, we examined the performance of these methods by evaluating their accuracy from the absolute difference between the empirical power and a desired or nominal power $(80 \%)$ denoted as D (Table 1 and S3). The better sample size method should have an empirical power close to the nominal power. We observed that the simulated power corresponding to $\mathrm{n}$ for all the methods is close to $80 \%$ power in many cases and the absolute mean of $D$ for M1 to M5 is $0.017,0.014,0.032,0.025$ and 0.014 , respectively. These results suggest that M1, M2 and M5 achieve a power much closer to the desired power (80\%) than M3 and M4 in terms of the accuracy. A further comparison of these methods was performed using a non-parametric paired Wilcox test and a parametric paired $t$ test for testing the significant difference of $D$ between two methods. The statistical test for the paired comparison of $D$ between M1 and M2, M1 and M5, M2 and M5, or M3 and M4 is not significant with the $p$-values $>0.2$ (Table 2). The results suggest that the M1, M2 and M5 methods perform better than the M3 and M4 methods in terms of the accuracy for achieving a desired power corresponding to the sample size. Moreover, Table S2 shows the $\mathrm{n}$ and the simulated power from the two new methods given $w=1, k=3 / 2$ and an $80 \%$ nominal power. We observe that a smaller sample size from the M1 is required when FC $<1$ and a larger $n$ is required when FC $>1$ compared to the M2 method. A further comparison among these methods will be discussed when we test multiple genes.

\subsection{Sample size and power estimation in the case of testing multiple genes with FDR-controlling}

The objective is to assess the performance of five methods for testing multiple genes via rejecting at least one null given a set of genes. First, we need to define the number of true negative and positive genes (DEGs) as well as the total number of genes in the test. In this simulation, the total number of genes $M$ per sample is set to be 10,000; the true DEG $M_{\mathrm{t}}$ is set to be 100 , and $M_{0}=M-M_{\mathrm{t}}$ is the number of genes not differentially expressed under $H_{0}$. The expected number of true DEGs and the empirical power corresponding to the desired power $80 \%$ is evaluated. The rest of the parameter settings including $u_{0}, \rho, w, \rho, \phi$ and $k$ are similar to those for testing a single gene. In addition, we set $w=1$ or 1.2 , and $k=\frac{1}{3}, 1$ or $\frac{3}{2}$, where a significance level $\alpha^{*}$ in (15) is calculated as 0.000425 , given a nominal FDR $(f=0.05)$. For a two-sided Wald test, a test statistic is based on $Z_{1-\alpha^{*} / 2}$ instead of $Z_{1-\alpha^{*}}$ for a one-sided Wald test for all the methods.

For each combination of these parameter settings, sample sizes are calculated using the formulas in (16-17) and the existing method in (18-19), given an 80\% nominal power and a two-sided test with $\alpha^{*}=0.000425$. The corresponding power given the calculated sample size is assessed using a two-sided Wald test statistic with $\alpha$ $=\alpha^{*}$ in (6). The FC between two treatment groups for the multiple corrections is considered to be significantly different when a two-sided $p$-value is $\leq \alpha^{*} / 2$, which is much smaller than the nominal significance level $\alpha=0.05$ for testing a single gene. The empirical power is obtained as the percentage of the number of times that the null hypothesis is rejected at the significance level $\alpha^{*}$ in the 5000 simulated data. Results for each combination of the desired parameters with different read depth $w$ and ratio of sample size $k$ in the case of balanced or imbalanced design will be discussed in detail.

The sample size and simulated power (in parentheses) for testing multiple genes in a combinational settings are listed in Table $3(w=1$ and $k=1)$, Table $5(w=1,1.2$ and $k=3 / 2)$ and $S 4(w=1,1.2$ and $k=2 / 3)$. We observed that the changing pattern of the sample size for different parameter values of $u_{0}$, $\phi$ and $\rho$ in the methods (Table 3,5 and S4) was similar to the pattern observed in the case of testing a single gene (Table 1, S1 and S2). However, given the same setting, a much larger sample size was required for testing multiple genes compared to testing a single gene, which is expected.

Table 3: Sample size and simulated power in parenthesis from five methods for testing 10,000 genes.

\begin{tabular}{|c|c|c|c|c|c|c|c|c|c|c|c|c|c|}
\hline \multirow[t]{2}{*}{$\rho$} & \multirow[t]{2}{*}{$\phi$} & \multirow[t]{2}{*}{$u_{0}$} & \multicolumn{5}{|c|}{$n_{0}(\%$ power $)$} & \multirow{2}{*}{$\begin{array}{r}\text { Average } \\
n_{0}^{*}\end{array}$} & \multicolumn{5}{|c|}{$\%$ power based on $n_{0}^{*}$} \\
\hline & & & M1 & M2 & M3 & M4 & M5 & & M1 & M2 & M3 & M4 & M5 \\
\hline \multirow[t]{11}{*}{0.5} & 0.1 & 1 & $127(79)$ & $159(79)$ & $124(81)$ & $120(80)$ & $124(81)$ & 131 & 80 & 65 & 84 & 86 & 83 \\
\hline & & 5 & $32(79)$ & $38(79)$ & $32(82)$ & $31(81)$ & $32(82)$ & 33 & 81 & 68 & 84 & 85 & 84 \\
\hline & & 10 & $20(78)$ & $23(78)$ & $21(83)$ & $20(80)$ & $20(80)$ & 21 & 82 & 72 & 83 & 85 & 84 \\
\hline & & 20 & $14(79)$ & $15(76)$ & $15(82)$ & $14(81)$ & $14(80)$ & 14 & 79 & 72 & 77 & 81 & 80 \\
\hline & 0.5 & 1 & $159(78)$ & $190(78)$ & $162(82)$ & $153(80)$ & $157(80)$ & 164 & 80 & 67 & 83 & 85 & 83 \\
\hline & & 5 & $63(78)$ & $70(78)$ & $71(84)$ & $64(81)$ & $64(80)$ & 66 & 81 & 75 & 79 & 82 & 82 \\
\hline & & 10 & $52(80)$ & $55(80)$ & $59(84)$ & $52(80)$ & $53(81)$ & 54 & 82 & 78 & 78 & 83 & 82 \\
\hline & & 20 & $46(80)$ & 47 (79) & $53(84)$ & $46(80)$ & $47(81)$ & 48 & 82 & 80 & 78 & 83 & 82 \\
\hline & 0.7 & 1 & 175 (79) & $206(78)$ & $181(82)$ & $169(80)$ & $174(81)$ & 181 & 80 & 69 & 82 & 84 & 83 \\
\hline & & 5 & 79 (79) & $86(80)$ & $90(84)$ & $80(81)$ & $81(81)$ & 83 & 83 & 77 & 79 & 84 & 83 \\
\hline & & 10 & 67 (79) & $71(80)$ & 78 (85) & $68(80)$ & $69(81)$ & 71 & 83 & 80 & 78 & 83 & 83 \\
\hline
\end{tabular}




\begin{tabular}{|c|c|c|c|c|c|c|c|c|c|c|c|c|c|}
\hline & & 20 & $61(79)$ & $63(79)$ & $72(85)$ & $62(79)$ & $63(80)$ & 64 & 82 & 80 & 76 & 82 & 81 \\
\hline \multirow[t]{12}{*}{0.7} & \multirow[t]{4}{*}{0.1} & 1 & 394 (79) & 445 (79) & 392 (81) & $389(81)$ & $396(81)$ & 403 & 81 & 72 & 83 & 83 & 82 \\
\hline & & 5 & $103(79)$ & $113(78)$ & $104(81)$ & $102(80)$ & $104(81)$ & 105 & 80 & 74 & 81 & 82 & 81 \\
\hline & & 10 & $66(78)$ & $72(79)$ & $68(80)$ & $66(79)$ & $67(79)$ & 68 & 79 & 75 & 80 & 81 & 80 \\
\hline & & 20 & $48(80)$ & $51(80)$ & $50(81)$ & $48(80)$ & $49(81)$ & 49 & 80 & 77 & 80 & 81 & 81 \\
\hline & \multirow[t]{4}{*}{0.5} & 1 & $514(79)$ & $565(79)$ & $518(80)$ & 509 (79) & $517(80)$ & 525 & 80 & 74 & 81 & 81 & 81 \\
\hline & & 5 & $223(79)$ & $233(80)$ & $230(81)$ & $223(80)$ & $225(81)$ & 227 & 81 & 78 & 81 & 82 & 81 \\
\hline & & 10 & $186(79)$ & $191(78)$ & $194(81)$ & $187(79)$ & $188(80)$ & 189 & 79 & 78 & 79 & 80 & 79 \\
\hline & & 20 & $168(80)$ & $171(80)$ & $176(81)$ & $169(80)$ & $170(80)$ & 171 & 81 & 80 & 80 & 81 & 81 \\
\hline & \multirow[t]{4}{*}{0.7} & 1 & $574(79)$ & $625(79)$ & $581(81)$ & $569(80)$ & $578(80)$ & 585 & 80 & 75 & 81 & 82 & 81 \\
\hline & & 5 & $282(79)$ & $293(78)$ & $293(80)$ & $283(80)$ & $285(80)$ & 287 & 80 & 77 & 79 & 80 & 80 \\
\hline & & 10 & $246(81)$ & $251(81)$ & $257(83)$ & $247(82)$ & $248(82)$ & 250 & 82 & 81 & 81 & 82 & 82 \\
\hline & & 20 & $228(80)$ & $231(80)$ & $239(81)$ & $229(80)$ & $230(80)$ & 231 & 81 & 80 & 80 & 81 & 81 \\
\hline \multirow[t]{12}{*}{1.5} & \multirow[t]{4}{*}{0.1} & 1 & $216(82)$ & $185(83)$ & $215(80)$ & $221(82)$ & $218(80)$ & 211 & 81 & 89 & 89 & 80 & 79 \\
\hline & & 5 & $62(82)$ & $56(82)$ & $63(81)$ & $65(84)$ & $63(81)$ & 62 & 82 & 87 & 43 & 81 & 80 \\
\hline & & 10 & $43(82)$ & $39(80)$ & $44(81)$ & $45(84)$ & $43(80)$ & 43 & 82 & 86 & 48 & 81 & 80 \\
\hline & & 20 & $33(81)$ & $31(80)$ & $34(80)$ & $35(83)$ & $34(81)$ & 33 & 81 & 83 & 52 & 80 & 80 \\
\hline & \multirow[t]{4}{*}{0.5} & 1 & 309 (81) & $278(82)$ & $314(81)$ & $321(83)$ & $311(80)$ & 307 & 81 & 87 & 87 & 80 & 80 \\
\hline & & 5 & $155(81)$ & $148(80)$ & $162(81)$ & $162(83)$ & $156(81)$ & 157 & 82 & 84 & 54 & 81 & 81 \\
\hline & & 10 & $135(80)$ & $132(80)$ & $143(82)$ & $142(83)$ & $137(81)$ & 138 & 82 & 83 & 56 & 82 & 82 \\
\hline & & 20 & $126(81)$ & $124(80)$ & $133(82)$ & $132(83)$ & $127(80)$ & 128 & 82 & 82 & 58 & 81 & 81 \\
\hline & \multirow[t]{4}{*}{0.7} & 1 & $355(82)$ & $324(82)$ & $364(82)$ & $369(84)$ & $358(80)$ & 354 & 82 & 86 & 86 & 81 & 80 \\
\hline & & 5 & $201(81)$ & $195(82)$ & $212(83)$ & $211(84)$ & $203(81)$ & 204 & 82 & 84 & 55 & 82 & 81 \\
\hline & & 10 & $182(81)$ & $178(80)$ & $192(82)$ & $191(83)$ & $184(81)$ & 185 & 81 & 83 & 57 & 81 & 81 \\
\hline & & 20 & $172(81)$ & $170(80)$ & $183(82)$ & $181(83)$ & $174(81)$ & 176 & 82 & 82 & 59 & 82 & 82 \\
\hline \multirow[t]{12}{*}{2} & \multirow[t]{4}{*}{0.1} & 1 & $67(83)$ & $51(84)$ & 67 (82) & $67(83)$ & $67(81)$ & 64 & 81 & 93 & 79 & 81 & 78 \\
\hline & & 5 & $20(84)$ & $17(84)$ & $21(84)$ & $21(85)$ & $20(82)$ & 20 & 84 & 91 & 81 & 83 & 82 \\
\hline & & 10 & $14(83)$ & $12(80)$ & $15(83)$ & $15(86)$ & $14(81)$ & 14 & 83 & 89 & 79 & 82 & 81 \\
\hline & & 20 & $11(81)$ & $10(80)$ & $12(82)$ & $12(85)$ & $12(85)$ & 11 & 81 & 85 & 76 & 80 & 80 \\
\hline & \multirow[t]{4}{*}{0.5} & 1 & $99(82)$ & $83(83)$ & $105(82)$ & $101(83)$ & $99(80)$ & 97 & 81 & 89 & 77 & 80 & 79 \\
\hline & & 5 & $52(82)$ & $48(81)$ & $59(84)$ & $54(84)$ & $53(82)$ & 53 & 83 & 87 & 78 & 82 & 82 \\
\hline & & 10 & $46(81)$ & $44(80)$ & $53(84)$ & $48(82)$ & $47(82)$ & 48 & 83 & 85 & 78 & 82 & 82 \\
\hline & & 20 & $43(82)$ & $42(81)$ & $51(86)$ & $45(84)$ & $44(82)$ & 45 & 84 & 85 & 78 & 84 & 84 \\
\hline & \multirow[t]{4}{*}{0.7} & 1 & $115(82)$ & $99(81)$ & $124(83)$ & $118(83)$ & $116(81)$ & 114 & 82 & 89 & 78 & 81 & 80 \\
\hline & & 5 & $67(81)$ & $64(81)$ & 78 (85) & $71(84)$ & $69(81)$ & 70 & 83 & 86 & 78 & 83 & 82 \\
\hline & & 10 & $61(81)$ & $60(81)$ & $72(84)$ & $65(83)$ & $63(82)$ & 64 & 83 & 85 & 77 & 83 & 82 \\
\hline & & 20 & $59(81)$ & $58(81)$ & $70(85)$ & $62(83)$ & $60(82)$ & 62 & 84 & 85 & 77 & 83 & 83 \\
\hline \multirow[t]{12}{*}{3} & \multirow[t]{4}{*}{0.1} & 1 & $24(87)$ & $15(84)$ & $24(85)$ & $23(85)$ & $23(83)$ & 22 & 81 & 97 & 79 & 82 & 80 \\
\hline & & 5 & $7(80)$ & $6(86)$ & $9(89)$ & $8(88)$ & $8(86)$ & 8 & 89 & 96 & 82 & 88 & 87 \\
\hline & & 10 & $5(78)$ & $4(76)$ & $7(91)$ & $6(89)$ & $6(88)$ & 6 & 90 & 95 & 81 & 89 & 88 \\
\hline & & 20 & $4(78)$ & $4(84)$ & $6(92)$ & $5(90)$ & $5(87)$ & 5 & 90 & 93 & 80 & 90 & 89 \\
\hline & \multirow[t]{4}{*}{0.5} & 1 & $37(84)$ & $28(82)$ & $43(87)$ & $43(87)$ & $37(82)$ & 38 & 85 & 94 & 78 & 85 & 83 \\
\hline & & 5 & $20(82)$ & $18(80)$ & $28(91)$ & $28(91)$ & $21(83)$ & 23 & 89 & 92 & 76 & 88 & 87 \\
\hline & & 10 & $18(81)$ & $17(80)$ & $26(92)$ & $26(92)$ & $19(82)$ & 21 & 90 & 91 & 77 & 89 & 88 \\
\hline & & 20 & $17(81)$ & $16(80)$ & $25(91)$ & $18(83)$ & $18(83)$ & 19 & 87 & 88 & 70 & 86 & 85 \\
\hline & \multirow[t]{4}{*}{0.7} & 1 & $43(83)$ & $35(83)$ & $52(87)$ & $44(84)$ & $43(80)$ & 43 & 83 & 92 & 73 & 82 & 80 \\
\hline & & 5 & $26(80)$ & $25(81)$ & $37(90)$ & $28(83)$ & $27(80)$ & 29 & 86 & 89 & 71 & 86 & 87 \\
\hline & & 10 & $24(81)$ & $23(80)$ & $35(90)$ & $26(84)$ & $25(81)$ & 27 & 87 & 88 & 70 & 85 & 85 \\
\hline & & 20 & $23(80)$ & $23(81)$ & 34 (91) & $25(84)$ & $24(80)$ & 26 & 88 & 88 & 69 & 86 & 86 \\
\hline
\end{tabular}

The parameter values are: fold change $(\rho)$, dispersion $(\phi)$ and mean reads in the control group $\left(u_{0}\right)$ given $w=1, \operatorname{FDR}=0.05, \alpha^{*}=0.000425$ and an $80 \%$ nominal power.

Since the empirical power varies for the corresponding sample size from different methods, we further evaluated these methods via a comparison of the simulated power for each method, which was calculated based on an average of the sample sizes $\left(n^{*}\right)$ for testing multiple genes in different settings (Table 3 ). The best method should achieve the highest power with a given $n^{*}$. With this approach, we observed that M1, M3 and M4 can achieve an empirical power higher than or close to $80 \%$ in most settings while M2 with FC < 1 and M3 with FC > 1 failed to achieve a power close to $80 \%$ in many settings. These results suggested that M2 with the parameters estimated from the MLE under $H_{0}$ for FC $<1$ and M3 for FC $>1$ could overestimate sample sizes for RNA-seq experiment design. However, the M2 at FC $>1$ outperforms other methods with greater power for a given $n^{*}$.

A further comparison of these methods was performed using a non-parametric Wilcoxon test and a parametric T-test statistic for testing the significance of the power from five methods given the average sample size $n^{*}$ (Table 3). The results from both test statistics (Table 3) indicated that the estimated power between any two 
of M1, M2, M4 and M5 is not significantly different with a $p$-value $>0.05$. However, the power from M3 is statistically different from that obtained by other methods with $p$-values $<0.0001$ (Table 4 ).

Table 4: The $p$-values obtained from a paired Wilcox test and t-test.

\begin{tabular}{crrrr}
\hline Wilcox test & M2 & M3 & M4 & M5 \\
\hline M1 & 0.4234 & $<0.0001$ & 0.3483 & 0.3653 \\
M2 & - & $<0.0001$ & 0.5359 & 0.2167 \\
M3 & & - & - & $<0.0001$ \\
M4 & & & & 0.0001 \\
T test & 0.8000 & $<0.0001$ & 0.6075 & 0.3187 \\
M1 & - & $<0.0001$ & $<0.0001$ & 0.4701 \\
M2 & & - & - & $<0.0001$ \\
M3 & & & 0.1051 \\
M4 & & & - \\
\hline
\end{tabular}

The test statistics are used for testing the significant difference of the simulated power estimated from the five methods.

Table 5 represents the sample size and simulated power in parentheses from our two methods in an imbalanced design $k=3 / 2$ with read depth $w=1$ and $w=1.2$. Given the same settings and $k=2 / 3$, the sample size and simulated power in parentheses are listed in Table S4 (Supporting Information in the supplementary file). We observed that the required sample size with unequal size factors given $w=1.2$ is slightly smaller than that with equal size factors (Table 5 and S4).

Table 5: Sample sizes and simulated power in parentheses from our two methods.

\begin{tabular}{|c|c|c|c|c|c|c|}
\hline \multirow[t]{2}{*}{$\rho$} & \multirow[t]{2}{*}{$\phi$} & \multirow[t]{2}{*}{$u_{0}$} & \multicolumn{2}{|c|}{$n_{0}$ (Simulated power) at $w=1$} & \multicolumn{2}{|c|}{$n_{0}$ (Simulated power) at $w=1.2$} \\
\hline & & & M1 & M2 & M1 & M2 \\
\hline \multirow[t]{12}{*}{0.5} & \multirow[t]{4}{*}{0.1} & 1 & $99(0.77)$ & $131(0.78)$ & $90(0.77)$ & $120(0.77)$ \\
\hline & & 5 & $25(0.77)$ & $31(0.76)$ & $23(0.76)$ & $29(0.76)$ \\
\hline & & 10 & $16(0.77)$ & $19(0.76)$ & $15(0.76)$ & $18(0.76)$ \\
\hline & & 20 & $11(0.75)$ & $13(0.76)$ & $11(0.77)$ & $12(0.78)$ \\
\hline & \multirow[t]{4}{*}{0.5} & 1 & $126(0.77)$ & $157(0.77)$ & $117(0.78)$ & $147(0.78)$ \\
\hline & & 5 & $52(0.78)$ & $58(0.78)$ & $50(0.79)$ & $56(0.78)$ \\
\hline & & 10 & $42(0.78)$ & $45(0.77)$ & $41(0.78)$ & $44(0.78)$ \\
\hline & & 20 & $38(0.79)$ & $39(0.80)$ & $37(0.80)$ & $39(0.79)$ \\
\hline & \multirow[t]{4}{*}{0.7} & 1 & $139(0.79)$ & $170(0.77)$ & $130(0.79)$ & $160(0.78)$ \\
\hline & & 5 & $65(0.80)$ & $71(0.79)$ & $63(0.80)$ & $69(0.80)$ \\
\hline & & 10 & $56(0.79)$ & $59(0.79)$ & $55(0.79)$ & $58(0.79)$ \\
\hline & & 20 & $51(0.79)$ & $53(0.80)$ & $50(0.80)$ & $52(0.79)$ \\
\hline \multirow[t]{12}{*}{0.7} & \multirow[t]{4}{*}{0.1} & 1 & $317(0.79)$ & $369(0.80)$ & $294(0.79)$ & $340(0.79)$ \\
\hline & & 5 & $83(0.79)$ & $94(0.79)$ & 79 (0.79) & $88(0.80)$ \\
\hline & & 10 & $54(0.79)$ & $53(0.79)$ & $52(0.79)$ & $57(0.79)$ \\
\hline & & 20 & $40(0.80)$ & $42(0.80)$ & $38(0.80)$ & $41(0.80)$ \\
\hline & \multirow[t]{4}{*}{0.5} & 1 & 417 (0.79) & $469(0.79)$ & $394(0.79)$ & $440(0.79)$ \\
\hline & & 5 & $183(0.80)$ & $194(0.79)$ & $179(0.79)$ & $188(0.79)$ \\
\hline & & 10 & $154(0.79)$ & $159(0.80)$ & $152(0.80)$ & $156(0.79)$ \\
\hline & & 20 & $139(0.79)$ & $142(0.80)$ & $138(0.79)$ & $141(0.79)$ \\
\hline & \multirow[t]{4}{*}{0.7} & 1 & 467 (0.79) & $519(0.79)$ & $443(0.79)$ & $490(0.79)$ \\
\hline & & 5 & $233(0.80)$ & $244(0.80)$ & $229(0.80)$ & $238(0.80)$ \\
\hline & & 10 & $204(0.79)$ & $209(0.79)$ & $202(0.79)$ & $206(0.79)$ \\
\hline & & 20 & $189(0.79)$ & $192(0.79)$ & $188(0.79)$ & $191(0.79)$ \\
\hline \multirow[t]{10}{*}{1.5} & \multirow[t]{4}{*}{0.1} & 1 & $187(0.83)$ & $155(0.83)$ & $178(0.83)$ & $145(0.83)$ \\
\hline & & 5 & $53(0.82)$ & $47(0.83)$ & $51(0.82)$ & $44(0.82)$ \\
\hline & & 10 & $36(0.81)$ & $33(0.81)$ & $35(0.82)$ & $32(0.81)$ \\
\hline & & 20 & $28(0.81)$ & $26(0.80)$ & $27(0.79)$ & $26(0.81)$ \\
\hline & \multirow[t]{4}{*}{0.5} & 1 & $264(0.82)$ & $233(0.82)$ & $255(0.82)$ & $222(0.82)$ \\
\hline & & 5 & $130(0.81)$ & $124(0.81)$ & $128(0.81)$ & $122(0.81)$ \\
\hline & & 10 & $113(0.81)$ & $110(0.81)$ & $112(0.81)$ & $109(0.81)$ \\
\hline & & 20 & $105(0.80)$ & $103(0.80)$ & $105(0.80)$ & $103(0.80)$ \\
\hline & \multirow[t]{2}{*}{0.7} & 1 & $303(0.81)$ & $271(0.81)$ & $294(0.82)$ & $261(0.81)$ \\
\hline & & 5 & $169(0.81)$ & $162(0.80)$ & $151(0.81)$ & $148(0.80)$ \\
\hline
\end{tabular}




\begin{tabular}{|c|c|c|c|c|c|c|}
\hline & & 10 & $152(0.81)$ & $149(0.81)$ & $209(0.81)$ & $206(0.81)$ \\
\hline & & 20 & $144(0.81)$ & $142(0.81)$ & $143(0.81)$ & $142(0.81)$ \\
\hline \multirow[t]{12}{*}{2.0} & 0.1 & 1 & $59(0.84)$ & $43(0.84)$ & $57(0.85)$ & $40(0.84)$ \\
\hline & & 5 & $17(0.83)$ & $14(0.84)$ & $17(0.85)$ & $13(0.82)$ \\
\hline & & 10 & $12(0.83)$ & $10(0.82)$ & $12(0.84)$ & $10(0.83)$ \\
\hline & & 20 & $9(0.93)$ & $8(0.85)$ & $9(0.84)$ & $8(0.79)$ \\
\hline & 0.5 & 1 & $86(0.83)$ & $70(0.83)$ & $84(0.84)$ & $67(0.83)$ \\
\hline & & 5 & $44(0.82)$ & $40(0.80)$ & $43(0.81)$ & $40(0.81)$ \\
\hline & & 10 & $38(0.80)$ & $37(0.81)$ & $38(0.81)$ & $36(0.79)$ \\
\hline & & 20 & $36(0.81)$ & $35(0.81)$ & $36(0.80)$ & $35(0.81)$ \\
\hline & 0.7 & 1 & $99(0.82)$ & $83(0.82)$ & $97(0.82)$ & $80(0.81)$ \\
\hline & & 5 & $57(0.81)$ & $54(0.80)$ & $56(0.81)$ & $53(0.81)$ \\
\hline & & 10 & $52(0.80)$ & $50(0.81)$ & $51(0.80)$ & $50(0.81)$ \\
\hline & & 20 & $49(0.81)$ & $48(0.81)$ & $49(0.81)$ & $48(0.81)$ \\
\hline \multirow[t]{12}{*}{3.0} & 0.1 & 1 & $22(0.87)$ & $13(0.87)$ & $21(0.89)$ & $12(0.85)$ \\
\hline & & 5 & $6(0.80)$ & $5(0.86)$ & $6(0.81)$ & $5(0.87)$ \\
\hline & & 10 & $5(0.88)$ & $4(0.87)$ & $5(0.88)$ & $4(0.88)$ \\
\hline & & 20 & $4(0.94)$ & $3(0.83)$ & $4(0.80)$ & $3(0.83)$ \\
\hline & 0.5 & 1 & $32(0.85)$ & $24(0.84)$ & $32(0.86)$ & $23(0.83)$ \\
\hline & & 5 & $17(0.82)$ & $15(0.80)$ & $17(0.83)$ & $15(0.81)$ \\
\hline & & 10 & $15(0.82)$ & $14(0.80)$ & $15(0.82)$ & $14(0.81)$ \\
\hline & & 20 & $14(0.83)$ & $14(0.79)$ & $14(0.83)$ & $14(0.79)$ \\
\hline & 0.7 & 1 & $38(0.84)$ & $29(0.83)$ & $37(0.84)$ & $28(0.81)$ \\
\hline & & 5 & $22(0.81)$ & $21(0.80)$ & $22(0.82)$ & $20(0.81)$ \\
\hline & & 10 & $20(0.82)$ & $19(0.81)$ & $20(0.81)$ & $19(0.81)$ \\
\hline & & 20 & $19(0.81)$ & $19(0.82)$ & $19(0.81)$ & $19(0.82)$ \\
\hline
\end{tabular}

$\alpha^{*}$ is the significance level at a desired FDR $=0.05$ for testing multiple genes. M1 and M2 are our methods.

The parameter value is imbalanced design $(k=3 / 2)$ given an $80 \%$ nominal power, and $\alpha^{*}=0.000425$.

\section{An example of real breast cancer data}

We used a real breast cancer study to illustrate how to estimate sample sizes using the formulas (13-14) for testing multiple genes. The RNA-seq dataset containing thirty-nine Triple Negative Breast Cancer (TNBC) primary tumors and twenty-one uninvolved breast tissue samples that were adjacent to TNBC primary tumors in .fastq format were downloaded from NCBI GEO (series ID GSE58135) (Varley et al., 2014). The raw sequencing files were mapped to the human hg19 reference genome using tophat2 (v2.0.13) with bowtie version (2.2.3.0). The mapped counts for 57,773 genes per sample were then extracted using HTSeq-scripts-count (version 2.7). After filtering the genes with zero counts in both treatment groups, sixty samples containing 36,762 genes were loaded into edgeR for estimating the NB parameters such as common dispersion and normalization factors called size factors in edgeR. The TMM (Trimmed Mean of M-value) normalization method from edgeR was used to estimate the size factors (Anders \& Huber, 2010). The mean size factors of $\bar{s}_{0}$ in the control group and $\bar{s}_{1}$ in the TNBC group are 0.936 and 1.065 , respectively. Thus, the ratio of the size factors $(w)$ is 1.14 . The estimated common dispersion $\phi$ is around 0.49 (Robinson \& Smyth, 2008).

We assumed that the top 500 of 36,762 genes (1.4\%) are prognostic and have the largest FC for up or downregulated genes. The minimum of average read counts among the 500 genes in the control group served as the pilot data with an estimated value as $u_{0} \approx 3$ (Li et al. 2013a; 2017). In addition, the sample sizes were estimated by setting $u_{0}=10,20$ or 50 for other genes. Suppose we want to set the nominal power to be $80 \%$, which indicates we want to identify 400 or more of the prognostic genes. Given the FDR at $f=0.05$ or 0.10 , and an $80 \%$ nominal power, we can set $m=36762, m_{1}=500, m_{0}=m-m_{1}$, and $r_{1}=400$ (the expected true DEG). The parameter $\rho_{g}(g=1, \ldots, 36,762)$ is assumed to be unknown. We set the mean counts in the control group to be $u_{0}=3$, 10,20 or 50 and FC to be $\rho_{g}=0.5,1.5,2$ or 3 with $\phi \approx 0.49$. With these settings, the new $\alpha^{*}=5.81 \times 10^{-4}$ or $1.23 \times$ $10^{-3}$ is obtained from (15) at a desired 0.05 or $0.10 \mathrm{FDR}$, respectively. Finally, the sample sizes are estimated by substituting $\alpha^{*}$ and an 80\% power into equations (10-11) (Table 6). Equal sample sizes were further estimated when assuming the top 500 genes are prognostic given 0.05 FDR (Table 6).

Table 6 reports unequal sample sizes $n_{0}$ in the control group and $n_{1}=k n_{0}$ in the TNBC group under different settings at $k=1$.9. We observed that the original RNA-seq experiment (Varley et al., 2014) with a minimum $n_{0}$ $=17$ and $n_{1}=33$ from method one (M1) can detect more than $80 \%$ of the prognostic genes at a two-sided FDR $(f=0.05)$ and the minimum $u_{0}=3$ if the desired FC for up-regulated genes is 3 or more. For an imbalanced 
experimental design, a new study needs 53 normal control and 101 cancer patients to achieve an $80 \%$ detection power if the desired FC for the down-regulated gene is 2 or more when using M1 method. With the same settings, a new study needs 44 normal control and 84 cancer patient samples for the up-regulated genes with a desired FC of 2 or more, which is fewer than the down-regulated genes. As the desired FC decreases to 1.5 or 1.2 , a new study in each group requires a larger number of patients in order to achieve an $80 \%$ nominal power for a two-sided test given a 0.05 FDR. Moreover, Table 6 shows that smaller sample sizes are required if the FDR increases from 0.05 to 0.10 for a less stringent test. Finally, Table 6 illustrates that a larger sample size $n_{0}$ is required for a balanced design than that for an unbalanced design.

Table 6: Sample sizes estimated our methods using the BC data.

\begin{tabular}{|c|c|c|c|c|c|c|c|}
\hline \multirow[t]{3}{*}{$\rho$} & \multirow[t]{3}{*}{$u_{0}$} & & & \multicolumn{2}{|c|}{ Unequal sample size $\left(n_{0}\right)$} & \multicolumn{2}{|c|}{ Equal sample size $\left(n_{0}\right)$} \\
\hline & & \multicolumn{2}{|c|}{$\mathrm{FDR}=0.05\left(\alpha^{*}=0.000,581\right)$} & \multicolumn{2}{|c|}{$\mathrm{FDR}=0.10\left(\alpha^{*}=0.001,226\right)$} & \multicolumn{2}{|c|}{$\mathrm{FDR}=0.05\left(\alpha^{*}=0.000,581\right)$} \\
\hline & & $n_{\mathrm{M} 1}$ & $n_{\mathrm{M} 2}$ & $n_{\mathrm{M} 1}$ & $n_{\mathrm{M} 2}$ & $n_{\mathrm{M} 1}$ & $n_{\mathrm{M} 2}$ \\
\hline \multirow[t]{4}{*}{0.5} & 3 & 53 & 63 & 48 & 57 & 72 & 83 \\
\hline & 10 & 36 & 39 & 32 & 35 & 48 & 51 \\
\hline & 20 & 32 & 34 & 29 & 30 & 43 & 44 \\
\hline & 50 & 30 & 31 & 27 & 28 & 40 & 40 \\
\hline \multirow[t]{4}{*}{0.8} & 3 & 469 & 490 & 425 & 443 & 618 & 643 \\
\hline & 10 & 333 & 340 & 302 & 308 & 438 & 445 \\
\hline & 20 & 304 & 308 & 276 & 278 & 399 & 403 \\
\hline & 50 & 287 & 288 & 260 & 261 & 376 & 378 \\
\hline \multirow[t]{4}{*}{1.2} & 3 & 667 & 639 & 604 & 579 & 859 & 834 \\
\hline & 10 & 489 & 480 & 443 & 435 & 636 & 629 \\
\hline & 20 & 451 & 447 & 408 & 404 & 588 & 585 \\
\hline & 50 & 428 & 426 & 387 & 386 & 560 & 558 \\
\hline \multirow[t]{4}{*}{1.5} & 3 & 132 & 121 & 120 & 110 & 168 & 158 \\
\hline & 10 & 98 & 95 & 89 & 86 & 127 & 124 \\
\hline & 20 & 91 & 89 & 82 & 81 & 118 & 117 \\
\hline & 50 & 86 & 86 & 78 & 78 & 113 & 112 \\
\hline \multirow[t]{4}{*}{2.0} & 3 & 44 & 39 & 40 & 35 & 56 & 51 \\
\hline & 10 & 33 & 32 & 30 & 29 & 43 & 41 \\
\hline & 20 & 31 & 30 & 28 & 27 & 40 & 39 \\
\hline & 50 & 29 & 29 & 27 & 26 & 39 & 38 \\
\hline \multirow[t]{4}{*}{3.0} & 3 & 17 & 14 & 16 & 13 & 21 & 19 \\
\hline & 10 & 13 & 12 & 12 & 11 & 17 & 16 \\
\hline & 20 & 12 & 12 & 11 & 11 & 16 & 15 \\
\hline & 50 & 12 & 12 & 11 & 10 & 15 & 15 \\
\hline
\end{tabular}

The parameter values are: imbalanced $(k=1.9)$ or balanced $(k=1)$ design given $w=1.14, \phi=0.49$, and an $80 \%$ nominal power.

\section{Discussion}

In this study, the M3 and M4 methods for sample size calculations are expended from an existing one-sided test to a two-sided test while comparing with our M1 and M3 methods. Since the null distribution of a one-sided Wald test with constrained MLE in a restricted parameter space is different from two-sided Wald test (Molenberghs \& Verbeke, 2007), it is difficult to compare the methods between one-sidedness and two-sidedness. Therefore, we chose the M3 methods from the current method for sample size calculations developed by Li et al. (2017), which was based on a one-sided Wald test with parameters that are estimated from the unconstrained MLE. More important, we use a two-sided Wald test statistic for the model parameter $b_{1}$ from a standard GLM to develop two novel sample size calculation methods (M1 and $M 2$ ) based on the DEG analysis inRNA-seq data, which is the major difference from the current methods for sample size calculations. We have incorporated a common dispersion parameter and the size factors via a log link function using a NB regression model which is extended from the study by Zhu and Lakkis (2014). In Zhu's study, the sample size calculation methods are only applied for COPD experimental design and not suitable for RNA-seq data. Moreover, the variance of the Wald test is estimated from the variance-covariance matrix with the parameters estimated from the MLE and CMLE which is different from the methods in Li et al. (2017). Since the dispersion parameter from MLE has no closed form, it is difficult to explicitly derive a sample size formula using a Wald test statistic. Therefore, in 
this study, we assumed that the dispersion parameter was a known constant while deriving the variance from the model parameters. This alternative choice has been commonly used by previous studies when deriving the sample size from a NB distribution (Zhu \& Lakkis, 2014; Li et al., 2017).

Previous sample size calculations for RNA-seq data using a NB distribution focus on a balanced design only. In this paper, sample size calculation methods are presented under a wide range of settings including the imbalanced design at a two-sided Wald test, unequal read depth and small sample sizes, which is applicable in many situations. Studies for DEG analysis of RNA-seq data commonly choose the cutoff value of the minimum average read counts per gene at one, five or ten. In this study, we used a wider range of gene read counts in the control group $(1,5,10$ or 20), in particular, a minimum of one read was used in the case of lowly expressed genes in the simulation. As we expected, a larger sample size for a lowly expressed gene is needed according to our simulation. However, a study requiring a large sample size to achieve a nominal power at $80 \%$ or higher is not feasible in practice due to the cost. As an alternative, the higher read depth sequencing may be chosen to increase the mean read counts in the sample, instead of directly increasing the sample size. Moreover, when testing multiple genes in the simulation, we arbitrarily chose 10,000 genes and 10\% true DEGs. In reality, the total number of detected genes, at least in RNA-seq data, could vary depending upon the read depth in each sequencing sample. Since the public methods for directly estimating sample sizes in an imbalanced design are not available, we focused on evaluating our methods and the three existing methods for a balanced design. In future studies, we will consider the case of multiple treatment groups, paired samples and comparing these methods for an imbalanced design as well.

In summary, sample sizes estimated from our two methods and the three existing methods vary in different parameter settings. The corresponding power of five methods assessed is close to the $80 \%$ nominal power in most cases. A comparison of our two new methods with the three existing methods based on a two-sided test shows that our closing form formula (M1) performs as well as the two existing methods M4 and M5. However, sample size formula (14) for the M4 and M5 methods is not in a closed form and needs to be solved via a numerical method. Although our two new methods are based on the Wald test statistic that utilizes a large sample property of the MLE, the proposed M1 and M4 perform similar to the M5 when comparing the accuracy of the power analysis. However, our M2 at FC $>1$ performs slightly better than the other four methods in terms of achieving a smaller sample size and the empirical power close to or greater than $80 \%$ when the minimum mean read counts is smaller than 20 in most settings (Table 6). This is due to the MLE of parameters obtained under the null hypothesis. Thus, the estimated $u_{0}$ in M2 is maximized when FC $>1$, which may result in under estimation of sample sizes. This observation is consistent with the results reported by Zhu and Lakkis (2014). Since it is not known whether gene expression levels in an RNA-seq experiment are up or down-regulated, we recommend to use the M1 method for quickly estimating sample sizes with a desired minimum fold change and minimum average read count. Therefore, our direct method (M1) can provide an alternative for researchers to quickly estimate sample sizes when designing a RNA-seq experiment for clinical research studies.

\section{Acknowledgements}

This work is part of a doctoral dissertation originally written by the first author under the supervision of Dr. Shesh Rai and Dr. Dongfeng Wu. We would like to thank the reviewers for the insightful comments and advice during the revision.

\section{Funding}

This work was support by National Institutes of Health, P20GM103436.

\section{Appendix A}

\section{Deriving Fisher's information matrix}

The first and second partial derivatives of the log likelihood function $(l)$ in equation (3) with respect to $\beta_{1}$ and $\beta_{2}$ are:

$$
\frac{\partial l}{\partial b_{0}}=\sum_{i=0}^{1} \sum_{j=1}^{n_{i}} y_{i j}-\sum_{i=0}^{1} \sum_{j=1}^{n_{i}}\left(y_{i j}+1 / \phi\right)\left(\frac{d_{i j}}{1+d_{i j}}\right),
$$




$$
\begin{gathered}
\frac{\partial l}{\partial b_{1}}=\sum_{i=0}^{1} \sum_{j=1}^{n_{i}} y_{i j}-\sum_{i=0}^{1} \sum_{j=1}^{n_{i}}\left(y_{i j}+1 / \phi\right)\left(\frac{x_{i} d_{i j}}{1+d_{i j}}\right), \\
\frac{\partial^{2} l}{\partial b_{0}^{2}}=-\sum_{i=0}^{1} \sum_{j=1}^{n_{i}}\left(y_{i j}+1 / \phi\right)\left[\frac{d_{i j}}{\left(1+d_{i j}\right)^{2}}\right], \\
\frac{\partial^{2} l}{\partial b_{0} \partial b_{1}}=-\sum_{i=0}^{1} \sum_{j=1}^{n_{i}}\left(y_{i j}+1 / \phi\right)\left[\frac{x_{i} d_{i j}}{\left(1+d_{i j}\right)^{2}}\right] \\
\frac{\partial^{2} l}{\partial b_{1}^{2}}=-\sum_{i=0}^{1} \sum_{j=1}^{n_{i}}\left(y_{i j}+1 / \phi\right)\left[\frac{x_{i}^{2} d_{i j}}{\left(1+d_{i j}\right)^{2}}\right]
\end{gathered}
$$

where $d_{i j}=\phi s_{i j} e^{b_{0}+b_{1} x_{i}}$ and assuming $\phi$ is constant.

The Fisher information matrix is defined as

$$
I_{n_{0}, n_{1}}\left(b_{0}, b_{1}\right)=E\left[-\frac{\partial^{2} l}{\partial\left(b_{0}, b_{1}\right)^{2}}\right]=\left[\begin{array}{ll}
\xi_{b_{0} b_{0}} & \xi_{b_{0 b_{1}}} \\
\xi_{b_{1} b_{0}} & \xi_{b_{1} b_{1}}
\end{array}\right] .
$$

From equations (22-24), we can obtain by simplification:

$$
\begin{aligned}
& \xi_{b_{0} b_{0}}=-E\left[\frac{\partial^{2} l}{\partial b_{0}^{2}}\right]=\frac{n_{0} \bar{s}_{0} e^{b_{0}}}{1+\phi \bar{s}_{0} e^{b_{0}}}+\frac{n_{1} \bar{s}_{1} e^{b_{0}+b_{1}}}{1+\phi \bar{s}_{1} e^{b_{0}+b_{1}}}, \\
& \xi_{\beta_{0 \beta_{1}}}=-E\left[\frac{\partial^{2} l}{\partial b_{0} \partial b_{1}}\right]=\frac{n_{1} \bar{s}_{1} e^{b_{0}+b_{1}}}{1+\phi \bar{s}_{1} e^{b_{0}+b_{1}}} \\
& \xi_{b_{1} b_{1}}=-E\left[\frac{\partial^{2} l}{\partial b_{1}^{2}}\right]=\frac{n_{1} \bar{s}_{1} e^{b_{0}+b_{1}}}{1+\phi \bar{s}_{1} e^{b_{0}+b_{1}}}
\end{aligned}
$$

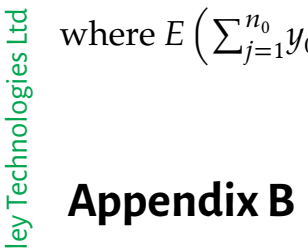

\section{Calculating MLEs of true expression parameter $\left(\gamma_{0}\right.$ and $\left.\gamma_{1}\right)$ under unrestricted and restricted scenarios}

The log likelihood function (l) with respect to $\gamma_{0}$ and $\gamma_{1}$ in equation (3) can be written as

$$
l=\sum_{i=0}^{1} \sum_{j=1}^{n_{i}}\left[\log \frac{\Gamma\left(\phi^{-1}+y_{i j}\right)}{\Gamma\left(\phi^{-1}\right) y_{i j} !}+y_{i j} \log \phi s_{i j} \gamma_{i}-\left(y_{i j}+\frac{1}{\phi}\right) \log \left(1+\phi s_{i j} \gamma_{i}\right)\right]
$$

Unrestricted scenario: The MLEs of $\left(\hat{\gamma}_{0}, \hat{\gamma}_{1}\right)^{\prime}$ can be estimated by setting the partial derivative of $l$ in B1 with the respect to $\left(\gamma_{0}, \gamma_{1}\right)^{\prime}$ to zero and then solving.

$$
\begin{gathered}
\frac{\partial l}{\partial \gamma_{0}}=\frac{1}{\gamma_{0}} \sum_{j=1}^{n_{0}} y_{0 j}-\frac{\phi \bar{s}_{0}}{1+\phi \bar{s}_{0} \gamma_{0}} \sum_{j=1}^{n_{0}} y_{0 j}-\frac{n_{0} \bar{s}_{0}}{1+\phi \bar{s}_{0} \gamma_{0}}, \\
\frac{\partial l}{\partial \gamma_{1}}=\frac{1}{\gamma_{1}} \sum_{j=1}^{n_{1}} y_{1 j}-\frac{\phi \bar{s}_{1}}{1+\phi \bar{s}_{1} \gamma_{1}} \sum_{j=1}^{n_{1}} y_{1 j}-\frac{n_{0} \bar{s}_{1}}{1+\phi \bar{s}_{1} \gamma_{1}},
\end{gathered}
$$




$$
\frac{\partial l}{\partial \gamma_{1}}=\frac{1}{\gamma_{1}} \sum_{j=1}^{n_{1}} y_{1 j}-\frac{\phi \bar{s}_{1}}{1+\phi \bar{s}_{1} \gamma_{1}} \sum_{j=1}^{n_{1}} y_{1 j}-\frac{n_{0} \bar{s}_{1}}{1+\phi \bar{s}_{1} \gamma_{1}}
$$

where setting $s_{0 j}=\bar{s}_{0}$ and $s_{1 j}=\bar{s}_{1}$ for simplification. Setting equations (27 and 28) to zero and solving, we get $\hat{\gamma}_{0}=\frac{\bar{y}_{0}}{\overline{\bar{s}}_{0}}, \hat{\gamma}_{1}=\frac{\bar{y}_{1}}{\overline{\bar{s}}_{1}}, \hat{u}_{0}=\bar{y}_{0}$ and $\hat{u}_{1}=\bar{y}_{1}$. Thus, the MLEs of $\left(\hat{b}_{0}, \hat{b}_{1}\right)$ are $\hat{b}_{0}=\log \left(\hat{\gamma}_{0}\right)=\log \left(\frac{\bar{y}_{0}}{\overline{\bar{s}}_{0}}\right)$, and $\hat{b}_{1}=\log \left(\frac{\hat{\gamma}_{1}}{\hat{\gamma}_{0}}\right)=$ $\log \left(\frac{\bar{y}_{1}}{w \bar{y}_{0}}\right)$.

For the constrained scenario $\left(H_{0}: \gamma_{0}=\gamma_{1}\right)$, the parameter $\gamma_{1}$ for $l$ in (25) is replaced by $\gamma_{0}$. We take the partial derivative of $l$ with respect to $\gamma_{0}$ and get:

$$
\frac{\partial l}{\partial \gamma_{0}}=\frac{1}{\gamma_{0}} Y_{0}-\frac{\phi \bar{s}_{0}}{1+\phi \bar{s}_{0} \gamma_{0}} Y_{0}-\frac{n_{0} \bar{s}_{0}}{1+\phi \bar{s}_{0} \gamma_{0}}+\frac{1}{\gamma_{0}} Y_{1}-\frac{\phi \bar{s}_{1}}{1+\phi \bar{s}_{1} \gamma_{0}} Y_{1}-\frac{n_{0} \bar{s}_{1}}{1+\phi \bar{s}_{1} \gamma_{0}}
$$

where $Y_{0}=\sum_{j=1}^{n_{0}} y_{0 j}$ and $Y_{1}=\sum_{j=1}^{n_{1}} y_{1 j}$.

For simplification, we set $\bar{s}_{0}=\bar{s}_{1}=\bar{s}$, and $\bar{s}=\frac{\bar{s}_{0}+\bar{s}_{1}}{2}=\frac{\bar{s}_{0}(1+w)}{2}$. Thus, we get $\tilde{\gamma}_{0}$ and $\tilde{u}_{0}$ by setting (29) to zero and solving

$$
\begin{aligned}
& \tilde{\gamma}_{0}=\frac{n_{0} \bar{y}_{0}+n_{1} \bar{y}_{1}}{\left(n_{0}+n_{1}\right) \bar{s}}=\frac{n_{0} \bar{s}_{0} \gamma_{0}+n_{1} \bar{s}_{1} \gamma_{1}}{\left(n_{0}+n_{1}\right) \frac{\overline{\bar{s}}_{0}(1+w)}{2}}=\frac{2(1+k w \rho) \gamma_{0}}{(1+k)(1+w)}, \\
& \tilde{u}_{0}=\bar{s}_{0} \tilde{\gamma}_{0}=\frac{2(1+k w \rho) u_{0}}{(1+k)(1+w)} .
\end{aligned}
$$

\section{References}

Aban, I. B., G. R. Cutter and N. Mavinga (2008): “Inferences and power analysis concerning two negative binomial distributions with an application to MRI lesion counts data," Comput. Stat. Data Anal., 53, 820-833.

Anders, S. and W. Huber (2010): “Differential expression analysis for sequence count data," Cenome Biol., 11, R106.

Benjamini, Y. and Y. Hochberg (1995): “Controlling the false discovery rate: a practical and powerful approach to multiple testing," J. R. Statist. Soc. B, 57, 289-300.

Fang, Z. and X. Cui (2011): “Design and validation issues in RNA-seq experiments," Brief. Bioinform. 12, 280-287.

Cuo, W., Q. Wang, Y. Zhan, X. Chen, Q. Yu, J. Zhang, Y. Wang, X. J. Xu and L. Zhu (2016): “Transcriptome sequencing uncovers a three-long noncoding RNA signature in predicting breast cancer survival," Sci. Rep., 6, 27931.

Keene, O. N., M. R. Jones, P. W. Lane and J. Anderson (2007): "Analysis of exacerbation rates in asthma and chronic obstructive pulmonary disease: example from the TRISTAN study," Pharm. Stat. 6, 89-97.

Li, C. I., P. F. Su, Y. Guo and Y. Shyr (2013a): "Sample size calculation for differential expression analysis of RNA-seq data under Poisson distribution," Int. J. Comput. Biol. Drug Des. 6, 358-375.

Li, C. I., P. F. Su and Y. Shyr (2013b): "Sample size calculation based on exact test for assessing differential expression analysis in RNA-seq data," BMC Bioinformatics, 14, 357.

Li, X., G. F. Cooper, Y. Shyr, D. Wu, E. C. Rouchka, R. S. Cill, T. E. O’Toole, G. N. Brock and S. N. Rai (2017): “Inference and sample size calculations based on statistical tests in a negative binomial distribution for differential gene expression in RNA-seq data," J. Biom. Biostat., 8 , 332.

Mi, G., Y. Di and D. W. Schafer (2015): “Coodness-of-fit tests and model diagnostics for negative binomial regression of RNA sequencing data," PLoS One 10, e0119254.

Molenberghs, G. and G. Verbeke (2007): “Likelihood ratio, score, and Wald tests in a constrained parameter space," Am. Stat., 61, 22-27.

Rajan, P., J. Stockley, I. M. Sudbery, J. T. Fleming, A. Hedley, G. Kalna, D. Sims, C. P. Ponting, A. Heger, C. N. Robson, R. M. McMenemin, I. D. Pedley and H. Y. Leung (2014): "Identification of a candidate prognostic gene signature by transcriptome analysis of matched pre- and post-treatment prostatic biopsies from patients with advanced prostate cancer," BMC Cancer, 14, 977.

Robinson, M. D. and A. Oshlack (2010): "A scaling normalization method for differential expression analysis of RNA-seq data," Cenome Biol., 11, R25.

Robinson, M. D. and G. K. Smyth (2008): "Small-sample estimation of negative binomial dispersion, with applications to SACE data," Biostatistics, 9, 321-332.

Smith, B. A., A. Sokolov, V. Uzunangelov, R. Baertsch, Y. Newton, K. Graim, C. Mathis, D. Cheng, J. M. Stuart and O. N. Witte (2015): “A basal stem cell signature identifies aggressive prostate cancer phenotypes," Proc. Natl. Acad. Sci. USA 112, E6544-6552.

Storey, J. D. (2002): “A direct approach to false discovery rates," ]. R. Stat. Soc. Ser. B 64, 479-498.

Varley, K. E., J. Gertz, B. S. Roberts, N. S. Davis, K. M. Bowling, M. K. Kirby, A. S. Nesmith, P. G. Oliver, W. E. Grizzle, A. Forero, D. J. Buchsbaum, A. F. LoBuglio and R. M. Myers (2014): “Recurrent read-through fusion transcripts in breast cancer," Breast Cancer Res. Treat., 146, $287-297$. 
Wright, H. L., H. B. Thomas, R. J. Moots and S. W. Edwards (2015): “Interferon gene expression signature in rheumatoid arthritis neutrophils correlates with a good response to TNFi therapy," Rheumatology (Oxford) 54, 188-193.

Wright, H. L., T. Cox, R. J. Moots and S. W. Edwards (2016): “Neutrophil biomarkers predict response to therapy with tumor necrosis factor inhibitors in rheumatoid arthritis," J. Leukoc. Biol., 101, 785-795.

Xu, L., J. Ziegelbauer, R. Wang, W. W. Wu, R. F. Shen, H. Juhl, Y. Zhang and A. Rosenberg (2016): “Distinct profiles for mitochondrial t-RNAs and small nucleolar RNAs in locally invasive and metastatic colorectal cancer," Clin. Cancer Res., 22, 773-784.

Yao, F., C. Zhang, W. Du, C. Liu and Y. Xu (2015): "Identification of gene-expression signatures and protein markers for breast cancer grading and staging," PLoS One, 10, e0138213.

Zhu, H. and H. Lakkis (2014): "Sample size calculation for comparing two negative binomial rates," Stat. Med., 33, 376-387.

Supplementary Material: The online version of this article offers supplementary material (DOI:https:/ /doi.org/10.1515/sagmb-2018-0021). 